\title{
Étude séroépidémiologique \\ de la toxoplasmose à la Guadeloupe \\ et à la Martinique
}

\author{
par J. TRIBOULEY, J. TRIBOULEY-DURET, M. APPRIOU, D. BALTZ \\ et R. PAUTRIZEL
}

Laboratoire d'Immunologie et de Biologie parasitaire, Université de Bordeaux II et Unité de Recherches sur l'Immunologie des Affections Parasitaires (I.N.S.E.R.M., U. 89), Domaine de Carreire, rue Camille-Saint-Saëns, F 33077 Bordeaux Cedex.

\section{Résumé.}

L'examen à la Guadeloupe et à la Martinique de 9950 sérums a mis en évidence l'importance et la précocité de l'infestation par le Toxoplasme : $56,9 \%$ de la population possède des anticorps fixant le complément, $65,6 \%$ des anticorps hémagglutinants et, dès les premières tranches d'âge, près de la moitié de la population se révèle infestée.

Bien que la consommation de viande insuffisamment cuite soit le plus souvent invoquée, ce mode de contamination ne semble pas devoir être incriminé à la Guadeloupe et à la Martinique où la population se nourrit traditionnellement de poisson et de viande très cuite. Par ailleurs la grande variabilité des taux d'infestation observés dans les différentes localités étudiées conduit à envisager l'intervention de facteurs climatiques favorisant la survie dans le milieu extérieur des oocystes issus de la multiplication sexuée du parasite.

\section{Summary.}

\section{Seroepidemiological survey on toxoplasmosis in Guadeloupe and Martinique.}

The examination of 9,950 serums in Guadeloupe and Martinique has revealed the importance and the precocity of infestation by Toxoplasma: $56,9 \%$ of the population has complement fixing antibodies, $65,6 \%$ haemagglutinating antibodies and from the very first age groups onwards, nearly half the population is found to be infested.

Reçu le 27 juillet 1977. 
Although the consumption of insufficien:ly cooked meat is most frequently invoked, it would seem that this manner of contamination should not be incriminated in Guadeloupe and Martinique where the population traditionally lives on fish and well-cooked meat. Moreover, the great variability in the rates of infestation observed in the différent localities studied leads one to envisage the intervention of climatic factors favoring the survival of the oocysts born of the sexed multiplication of the parasite in the external environment.

L'infection par le Toxoplasme est sans conteste la plus répandue des infestations à Protozoaires; on la trouve sur tous les continents et dans tous les pays mais sa fréquence est très variable suivant les régions. Un certain nombre de cas cliniques ont̂ été rapportés récemment à la Martinique (4) tandis que les premières recherches que nous avions effectuées à la Guadeloupe laissaient entrevoir la précocité et la fréquence de l'infestation inapparente (13). C'est afin de préciser ces résultats préliminaires que nous avons entrepris une enquête séro-épidémiologique de la toxoplasmose dans différentes régions géographiques de la Guadeloupe et de la Martinique.

\section{Matériel et techniques}

\section{Les sérums étudiés.}

Prélevés dans différentes communes de la Guadeloupe et de la Martinique avec l'accord et l'aide des autorités locales, les sérums ont été immédiatement congelés à $-20{ }^{\circ} \mathrm{C}$ et acheminés par bateau (conservés en chambre froide) à Bordeaux; 9950 sérums ont pu être examinés.

\section{Techniques sérologiques mises en œuvre.}

\section{La préparation de l'antigène.}

L'antigène a été préparé à partir de Toxoplasmes récoltés chez la Souris (souche Westphal). Afin d'obtenir une suspension riche en parasites, on injecte simultanément à l'animal par voie intra-péritonéale une suspension de Toxoplasmes et de cellules sarcomateuses (sarcome TG 180 de la Souris) servant de support à la prolifération du parasite [1]. L'inoculation à l'animal sous un volume de $0,5 \mathrm{ml}$ de $2.10^{6}$ Toxoplasmes et $10^{7}$ cellules sarcomateuses permet d'obtenir au $4^{\circ}$ jour de l'infestation une ascite pratiquement dépourvue de cellules et très riche en Toxoplasmes libres. La suspension de parasites ainsi obtenue est lavée à plusieurs reprises à $+4{ }^{\circ} \mathrm{C}$ et le culot de Toxoplasmes est conservé à $-25^{\circ} \mathrm{C}$.

L'extraction de l'antigène est effectuée suivant la méthode de Pettersen (14) : extraction alcaline par le carbonate de sodium qui se montre plus efficace que la méthode habituelle par congélation et décongélation. Schématiquement, le culot remis en suspension dans l'eau physiologiquement à raison de $1 \mathrm{~g}$ pour $5 \mathrm{ml}$ est additionné de $\mathrm{CO}_{3} \mathrm{Na}_{2}$ à raison de $16 \mathrm{mg} / \mathrm{ml}$ de suspension. Après agitation pendant 10 minutes, l'extrait dilué au $1 / 2$ en eau distillée est ramené à la neutralité par $\mathrm{HCl} \mathrm{N}$. Il est ensuite centrifugé et le surnageant recueilli est lyophilisé. 
- Réactions sérologiques utilisées.

Les sérums examinés sont testés par deux techniques: réaction de fixation du complément et réaction d'hémagglutination passive.

La réaction de fixation du complément est réalisée en microplaques en polystyrène à cupules à fond rond suivant une microméthode décrite par ailleurs (21).

La réaction d'hémagglutination passive est effectuée suivant une technique utilisant le glutaraldéhyde comme agent de couplage $(2,18,19,20)$. La sensibilisation des hématies est réalisée en solution tampon phosphate $0,15 \mathrm{M} \mathrm{pH}=7,2$, en mélangeant 0,5 volume de solution antigénique avec le culot globulaire correspondant à 2,5 volumes d'une suspension d'hématies de mouton à $2,5 \%$, en présence de 0,3 volume d'une solution aqueuse de glutaraldéhyde à $2,5 \%$. Les réactifs sont maintenus en contact pendant 45 minutes à la température du laboratoire en agitant fréquemment. Les hématies sont ensuite lavées en solution phosphate et remises en suspension dans 5 volumes d'une solution d'albumine humaine à $0,2 \%$. La réaction est effectuée en microplaques sur le sérum préalablement saturé en hémagglutines naturelles anti-mouton et dilué en progression géométrique de raison $1 / 2$ à partir d'une dilution initiale au 1/10. On ajoute 1 goutte de la suspension d'hématies sensibilisées mesurée à la pipette de Kline à $0,05 \mathrm{ml}$ de chaque dilution. La lecture est effectuée le lendemain.

\section{Résultats}

Etude comparée des résultats obtenus par la réaction de fixation du complément et la réaction d'hémagglutination passive.

Le taux moyen de sérums positifs obtenu à l'aide de la réaction de fixation du complément est de $56,9 \%$; le taux moyen obtenu à l'aide de la réaction d'hémagglutination passive est de $65,6 \%$ donc nettement supérieur. La corrélation entre les deux tests est cependant excellente comme le montre la comparaison des pourcentages obtenus dans les différentes localités étudiées (fig. 1).

Les taux en anticorps obtenus par la réaction de fixation du complément s'étalent du $1 / 1$ au $1 / 1024$, ceux obtenus par la réaction d'hémagglutination passive s'étalent du $1 / 10$ au $1 / 40960$; les résultats du tableau 1 montrent la bonne corrélation entre les deux tests bien que des discordances individuelles soient observées.

\section{Evolution des anticorps en fonction de l'âge.}

La détermination des pourcentages de sujets présentant des anticorps dans les tranches diâge comprises entre 4 et 74 ans montre que le taux d'infestation est marqué dès les premières tranches d'âge où il atteint $40 \%$ par la réaction de fixation du complément et $50 \%$ par la réaction d'hémagglutination passive. Il s'accroît rapidement jusqu'à 24 ans et se stabilise alors à des valeurs voisines de $70 \%$ pour la réaction de fixation du complément et $80 \%$ pour la réaction d'hémagglutination 


\begin{tabular}{|c|c|c|c|c|c|c|c|c|c|c|c|c|c|}
\hline & & - & $m$ & $m$ & - & $m$ & & & & & & & $\begin{array}{l}\stackrel{8}{\circ} \\
\text { \&े } \\
=\end{array}$ \\
\hline-1 & & N & 0 & $\forall$ & $m$ & $N$ & $N$ & $m$ & - & & & & 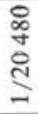 \\
\hline & - & $\forall$ & $\stackrel{\bullet}{\circ}$ & t & $m$ & $N$ & $N$ & & & & $m$ & & 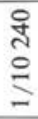 \\
\hline- & -1 & 으 & సె & $=$ & 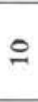 & $=$ & $n$ & $N$ & - & - & $N$ & & $\begin{array}{l}\text { స్ } \\
\text { ป }\end{array}$ \\
\hline & & $r$ & $\stackrel{2}{2}$ & $\exists$ & \pm & 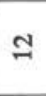 & $\infty$ & 6 & $m$ & & - & & $\begin{array}{l}: \\
\stackrel{0}{0} \\
\stackrel{2}{N}\end{array}$ \\
\hline & $\mathrm{N}$ & $a$ & 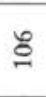 & $\approx$ & ఖ & 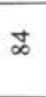 & $\approx$ & ले & ते & $\infty$ & กิ & $\forall$ & 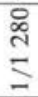 \\
\hline & $\mathrm{N}$ & $\forall$ & तి & $\cong$ & $\stackrel{\cong}{=}$ & 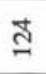 & $\stackrel{8}{\circ}$ & $\stackrel{\Omega}{\Xi}$ & $f$ & 6 & ஜ & 0 & ? \\
\hline- & $N$ & $N$ & $\Rightarrow$ & $\stackrel{\infty}{\sim}$ & $\tilde{\mathrm{I}}$ & $\stackrel{\infty}{\sim}$ & ¿̊. & $\stackrel{\infty}{\stackrel{\infty}{-1}}$ & $\infty$ & i & $\hat{6}$ & $\stackrel{0}{0}$ & స్లి \\
\hline & $\mathrm{m}$ & & 0 & $\stackrel{\circ}{\circ}$ & ฉి & ڤ̊ & $\stackrel{\infty}{\tilde{\pi}}$ & సે & ర్ర & in & ลิ & $g$ & $\stackrel{8}{\Xi}$ \\
\hline & & $N$ & $m$ & 0 & $r$ & $\ddot{\sim}$ & हे & 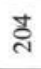 & 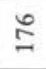 & $\bar{a}$ & $\tilde{\Xi}$ & త & $\stackrel{\infty}{=}$ \\
\hline & & $\mathrm{N}$ & $N$ & $n$ & 오 & $\vec{\sim}$ & \& & ర్ల & § & $\stackrel{\Xi}{0}$ & $\stackrel{0}{=}$ & 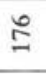 & $\stackrel{8}{\stackrel{9}{2}}$ \\
\hline & & & $\mathrm{N}$ & - & - & 0 & $\cong$ & $\Sigma$ & $\tilde{0}$ & $\bar{F}$ & $\approx$ & $\stackrel{\Xi}{\sim}$ & ్ㅗ \\
\hline & & & & - & & 0 & $a$ & $\hat{\lambda}$ & ন & $\bar{\infty}$ & $\infty$ & $\stackrel{\text { I }}{n}$ & $\stackrel{0}{\Xi}$ \\
\hline & & & & & - & & - & $\forall$ & $m$ & $\infty$ & $m$ & $\approx$ & 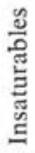 \\
\hline & & & $N$ & - & $m$ & $m$ & r & ন & $\vec{\sim}$ & $r$ & $\approx$ & 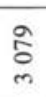 & 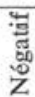 \\
\hline$\stackrel{\text { ¿ }}{\stackrel{\Xi}{二}}$ & $\begin{array}{c}\vdots \\
\vdots \\
\vdots \\
\vdots \\
\stackrel{2}{\Xi}\end{array}$ & 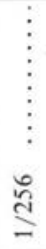 & $\begin{array}{c}\vdots \\
\vdots \\
\stackrel{\infty}{\simeq}\end{array}$ & $\begin{array}{c}\vdots \\
\vdots \\
\vdots \\
\stackrel{+}{=}\end{array}$ & $\begin{array}{c}\vdots \\
\vdots \\
\vdots \\
\vdots \\
\text { กี }\end{array}$ & $\begin{array}{l}\vdots \\
\vdots \\
\vdots \\
\vdots \\
ٍ\end{array}$ & $\stackrel{\infty}{=}$ & 巳 & $\stackrel{\sim}{\mathrm{N}}$ & $\begin{array}{l}\vdots \\
\vdots \\
\vdots \\
\vdots \\
\vdots\end{array}$ & 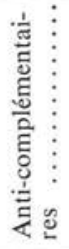 & 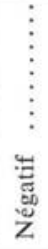 & \\
\hline
\end{tabular}




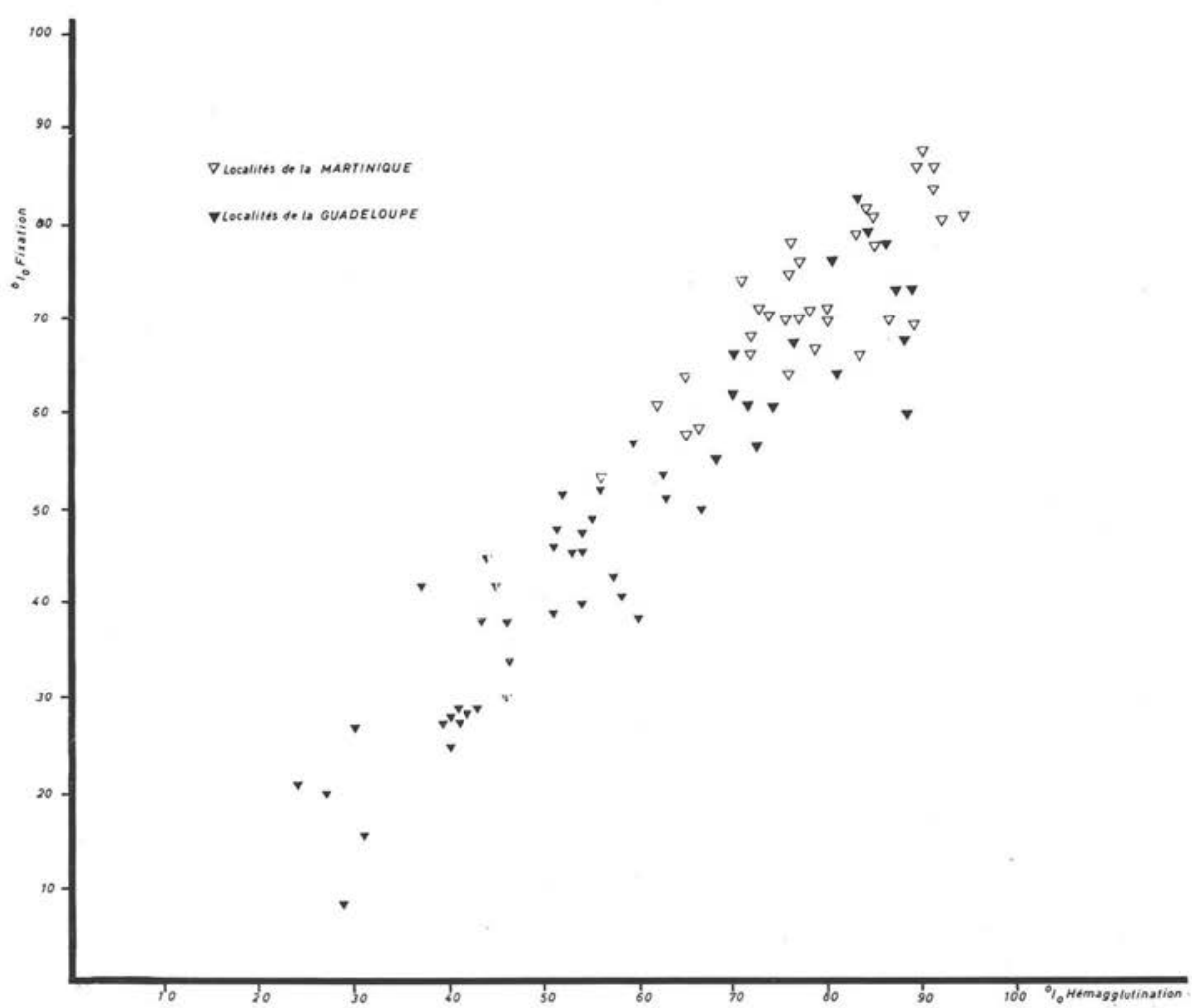

F1G. 1. - Etude comparée des pourcentages de positivité obtenus par la réaction de fixation du complément et la réaction d'hémagglutination passive dans différentes localités de la Guadeloupe et de la Martinique.

passive (fig. 2). Parallèlement le titre moyen des anticorps est maximum pour les premières tranches d'âge et il diminue rapidement pour se stabiliser ensuite (fig. 3).

\section{Pourcentages de positivité dans les différentes localités étudiées.}

Une grande variabilité est observée suivant les localités étudiées, les pourcentages relevés allant de 10 à près de $90 \%$ (fig. 1). D'une manière générale, les chiffres relevés en Martinique sont plus élevés que ceux de la Guadeloupe: c'est ainsi que le pourcentage global d'infestation en Martinique avoisine $73 \%$ par la réaction de fixation du complément et $80 \%$ par la réaction d'hémagglutination passive qu'en Guadeloupe les chiffres obtenus sont respectivement de $42,7 \%$ et $53,3 \%$. 


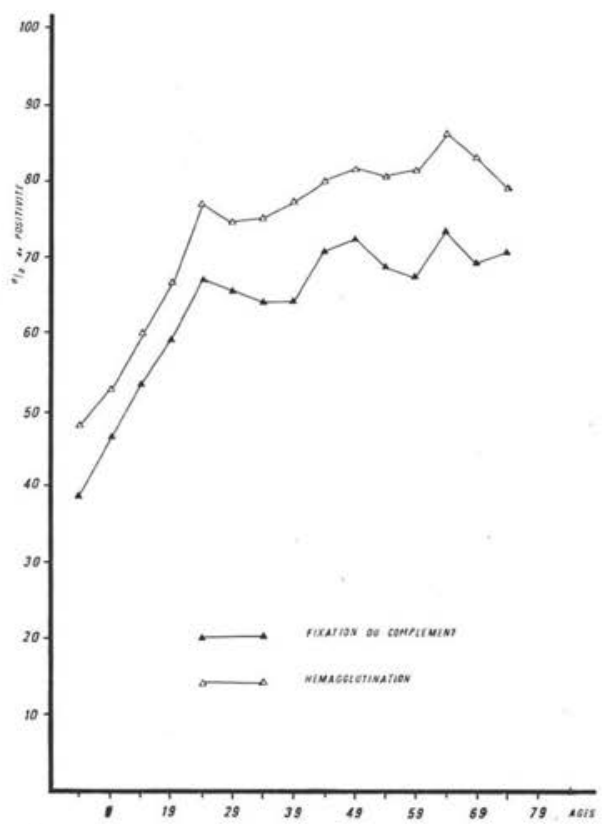

FIG. 2. - Evolution du taux d'infestation en fonction de l'âge.

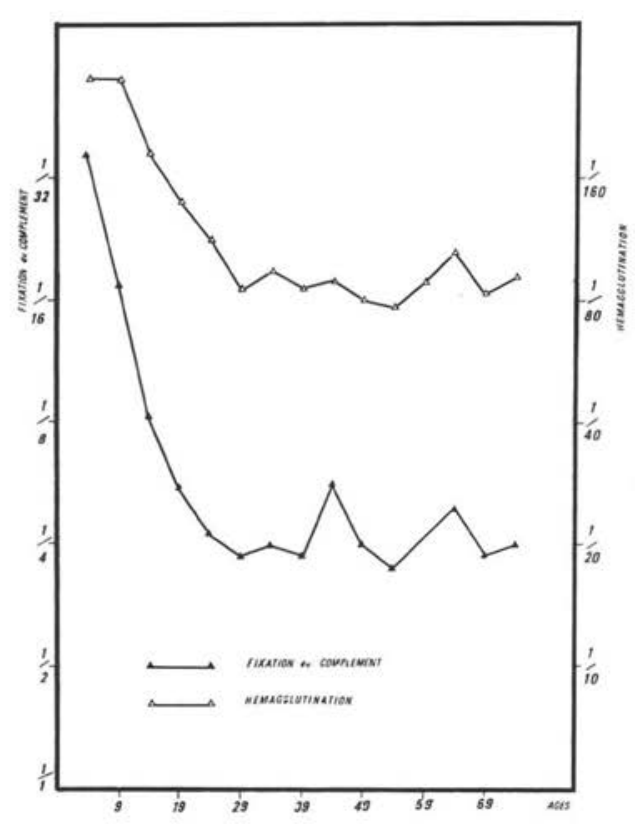

FIg. 3. - Evolution du taux des anticorps en fonction de l'âge.

Plus intéressantes à considérer sont les variations observées dans chacune des deux îles. A la Martinique, les localités les plus touchées se situent au Nord d'une ligne Robert-Fort de France où les pourcentages d'infestation sont de $75,4 \%$ (réaction de fixation du complément) et $81,1 \%$ (hémagglutination passive) tandis que dans le Sud les chiffres observés sont recpectivement de 64 et $67 \%$ (carte 1). La comparaison des pourcentages par le test du $\mathrm{X}^{2}$ montre que ces différences sont hautement significatives $(p<0,001)$. De même à la Guadeloupe, les différences notables peuvent être notées (carte 2) : c'est ainsi qu'à la Grande-Terre les pourcentages observés sont de $31,5 \%$ et $41,5 \%$; à la Basse-Terre, les chiffres relevés sont beaucoup plus élevés tout particulièrement dans les localités du Sud situées dans une zone allant de Baillif et Capesterre où les pourcentages observés sont de $64,6 \%$ et $76,6 \%$. Les valeurs relevées pour la côte sous le vent (de Baillif à Deshaies) sont de $31,5 \%$ et $41,5 \%$ tandis que pour la côte au vent des pourcentages de $41,6 \%$ et $50,8 \%$ sont notés. L'étude du $X_{2}$ montre que les différences observées entre ces différentes valeurs sont également significatives $(p<0,001)$. 


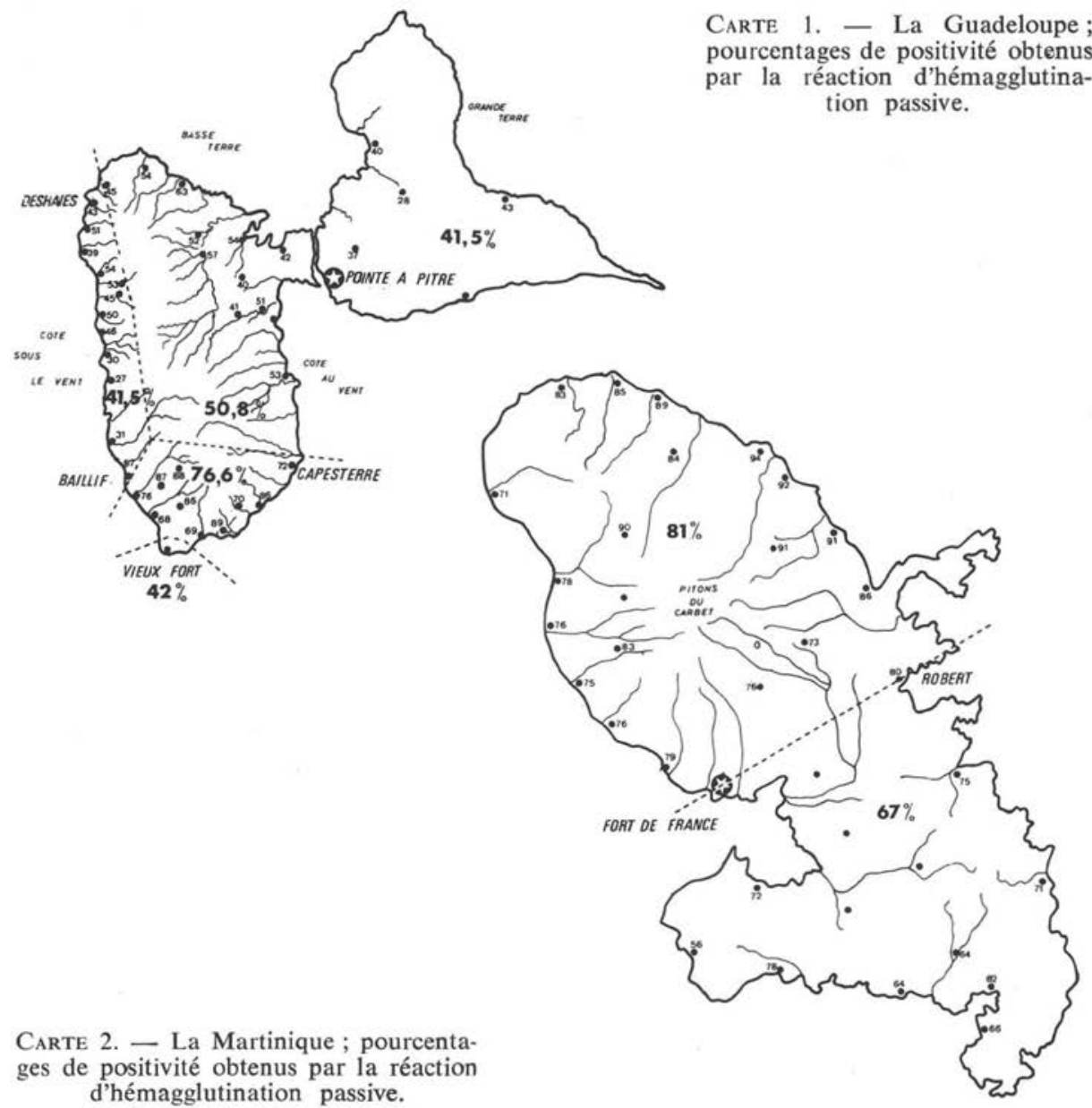

\section{Discussion}

Intérêt des réactions de fixation du complément et d'hémagglutination passive appliquées à l'épidémiologie de la toxoplasmose.

Lorsque la réaction est effectuée avec un extrait antigénique convenable, les anticorps fixant le complément apparaissent précocement au cours de l'infestation et peuvent ainsi permettre le diagnostic des toxoplasmoses en évolution. Il est fréquent d'observer à ce stade de l'infestation des taux élevés en anticorps fixant le complément alors que la réaction d'hémagglutination passive est encore négative ou faible- 
ment positive; une telle dissociation peut être considérée comme très évocatrice d'une infestation récente. D'autre part, la réaction de fixation pratiquée avec l'extrait antigénique utilisé au cours de ce travail met en évidence la longue persistance des anticorps apparus puisque le pourcentage de positivité maximum dès l'âge de 24 ans se maintient en plateau jusqu'aux tranches d'âges les plus avancées (fig. 2). Mais il faut signaler qu'avec d'autres préparations antigéniques les anticorps fixant le complément ne sont décelés que de façon beaucoup plus transitoire, ce qui restreint d'autant leur intérêt sur le plan épidémiologique (13).

La réaction d'hémagglutination passive est plus sensible que la réaction de fixation du complément. De plus, elle a l'avantage d'une plus grande simplicité d'exécution et peut facilement être automatisée, ce qui en fait un test particulièrement adapté aux enquêtes épidémiologiques. La phase préalable de fixation de l'antigène sur les hématies est la plus délicate, mais elle est simplifiée par l'utilisation du glutaraldéhyde comme agent de couplage; les hématies ainsi sensibilisées peuvent être éventuellement lyophilisées et conservées plusieurs mois. Enfin la réaction d'hémagglutination passive est relativement lente à se positiver, mais une fois apparus, les anticorps hémagglutinants persistent de longues années, sinon indéfiniment, ce qui est encore un avantage pour des études épidémiologiques. En raison de sa simp̧licité d'exécution, d'une automatisation facile et de la longue persistance des anticorps quel que soit le mode de préparation de l'antigène, la réaction d'hémagglutination passive apparaît donc comme la méthode de choix pour la réalisation des enquêtes séro-épidémiologiques.

\section{Les facteurs favorisant l'infestation à la Guadeloupe et à la Martinique.}

Les résultats présentés montrent la précocité et l'intensité de l'infestation à la Guadeloupe et à la Martinique. La toxoplasmose est une parasitose répandue dans le monde entier mais dont la fréquence est extrêmement variable suivant les régions: c'est ainsi que Feldmann rapporte $68 \%$ de sujets infestés à Tahiti tandis que chez les Esquimaux ce pourcentage est nul (7). A Paris, Desmonts note un taux d'infestation voisin de $90 \%$ chez les sujets de plus de 30 ans (5). Les causes de cette variabilité ne sont pas connues de façon certaine $(8,16)$. La consommation de viande insuffisamment cuite est le plus souvent invoquée pour expliquer les taux d'infestation élevés observés en France (5) ; effectivement les kystes du parasite peuvent être mis en évidence au niveau des muscles de diverses espèces animales (26). Ce serait pour la même raison que les populations créoles de Guyane française présenteraient un taux d'infestation supérieur à celui des Noirs et des Indiens (17) bien que dans ce cas la densité des populations étudiées puisse aussi jouer un rôle important. Les habitudes alimentaires sont également invoquées pour expliquer les taux d'infestations importants qui ont été récemment observés au Mali et au Togo, tout particulièrement en zone rurale où la contamination est très précoce $(11,15)$. Cependant l'ingestion de viande crue ou peu cuite ne peut être incriminée à la Guadeloupe et à la Martinique où la population se nourrit traditionnellement de poisson et de viande 
très cuite et ces habitudes alimentaires se sont, semble-t-il, maintenues jusqu'à l'époque actuelle. Une constatation analogue a été faite pour les îles du Pacifique où des taux d'infestation élevés sont observés bien que les protéines animales soient consommées en faible quantité et proviennent principalement d'espèces marines (22). D'autres modes de contamination que la consommation de viande crue ou insuffisamment cuite doivent donc être envisagés; l'infestation de diverses espèces animales herbivores est d'ailleurs un fait bien connu. Il est intéressant de remarquer que tant à la Guadeloupe qu'à la Martinique, les régions les plus sèches montrent des taux d'infestation moindres ; c'est ainsi qu'à la Martinique les localités situées au Sud d'une ligne Robert-Fort-de-France sont moins touchées que celles du Nord, région montagneuse au climat plus humide. A la Guadeloupe, la même opposition est retrouvée entre la Grande-Terre, plateau calcaire au climat sec où les pourcentages d'infestation ne sont que de $41,5 \%$ et le Sud de la Basse-Terre où les pourcentages relevés sont de 76,6\% (exception faite pour la localité de Vieux-Fort située sur un petit plateau calcaire et $\mathrm{sec}$ où les pourcentages de positivité n'atteignent que $42 \%$ ). Les valeurs retrouvées au niveau de la côte sous le vent de la Basse-Terre, relativement sèche $(41,5 \%)$ et celles de la côte au vent, plus humide $(50,8 \%)$ sont également différentes. Il semblerait donc que des facteurs climatiques puissent agir sur l'infestation par le Toxoplasme. Des constatations semblables ont été effectuées à l'île de la Réunion où les taux d'infestation sont également plus importants sur la côte au vent (3). Walls et Kagan signalent aux U.S.A. et au Brésil des variations des taux d'infestation en fonction des conditions climatiques; les régions au climat humide ayant des taux d'infestation plus élevés $(23,24)$. L'influence des conditions climatiques sur les taux d'infestation conduit naturellement à envisager le rôle que pourrait jouer dans la transmission un stade du parasite susceptible de se maintenir dans le milieu extérieur. L'hypothèse de la transmission par l'intermédiaire des œufs de Nématodes a été d'abord envisagée à la suite des travaux d'Hutchinson (9), mais à l'heure actuelle il est démontré que le Toxoplasme est en mesure d'évoluer chez le Chat par des processus de multiplication asexuée et sexuée $(10,26)$. Les oocystes issus de la multiplication sexuée qui se déroule dans l'intestin sont éliminés dans le milieu extérieur. Leur pouvoir infestant a été démontré $(25)$; leur résistance a été étudiée vis-à-vis de divers agents physiques et chimiques et il a en particulier été montré que leur pouvoir infestant se maintenait d'autant plus longtemps que le degré d'hygrométrie était plus élevé $(6,26)$. Toutes ces données sont donc en faveur de l'existence d'un réservoir tellurique à partir duquel l'homme et les animaux sont susceptibles de s'infester. Mais des inconnues persistent et il est difficile de penser que le Chat soit la seule espèce responsable de la dissémination des oocystes infestants (27).

\section{Bibliographie}

1. Ardoin (P.), Couzineau (P.) et Baufine-Ducroce (H.), 1967. - Sur l'utilisation du sarcome TG 180 pour l'obtention d'une suspension riche en Toxoplasma gondii extracellulaires. C.R. Soc. Biol (Paris), 161, 117-119. 
2. Avrameas (S.), Taudou (B.) et Chuilon (S.), 1969. - Glutaraldehyde, cyanuric chloride and tetra azotized o-dianisidine as coupling reagents in the passive hemagglutinating test. Immunochemistry, 6, 67.

3. Biguet (J.), Gérard (G.) et Bouthemy (F.), 1974. - La toxoplasmose à la Réunion. Bourbon Méd., 38, 3-15.

4. Chanol (M.-A.), 1974. - La toxoplasmose à la Martinique. Thèse médecine Bordeaux.

5. Desmont (G.), 1962. - Epidémiologie de la toxoplasmose. Rev. Hyg., 10, 201-217.

6. Dubey (J.-P), Miller (N.-L.) et Frenckel (J.-K.), 1970. - Characterization of the new fecal form of Toxoplasma gondii. J. Parasitol., 56, 447-456.

7. Feldman (H. A.) et Miller (L. T.), 1956. - Serological study of toxoplasmosis prevalence. Am. J. Hyg., 64, 320-335.

8. Garin (J.-P.), Baylet (R.), Despeignes (J.), Kien Tuang (T.), Rioche (M.) et Correa (P.), 1971. - Recheiches épidémiologiques sur la toxoplasmose humaine et animale au Sénégal. Med. Afr. Noire, 18, 751-753.

9. Hutchinson (W.M.), 1967. - The Nematode transmission of Toxoplasma gondii. Trans. R. Soc. trop. Med. Hyg., 61, 80-89.

10. Hutchinson (W. M.), Dunachie (J. F.), Sirm (J. Ch.) et Work (K.), 1970. - Coccidianlike Nature of Toxoplasma gondii. Br. med. J., 1, 142-144.

11. Lapierre (J.), Vover (V.-M.), Amedonne (A.), Holler (C.), Tourte-Schaeffer (C.) et Agвo (N.), 1976. - - Première enquête séro-immunologique sur la toxoplasmose au Togo. Bull. Soc. Pathol. exot., 69, 466-470.

12. Marc (Y.), Tribouley-Duret (J.) et Pautrizel (R.), 1975. - Etude comparative de plusieurs techniques séro-immunologiques appliquées au diagnostic de la toxoplasmose. Colloque sur la sérologie de l'infection toxoplasmique en particulier à son début: méthodes et interprétation des résultats, Lyon, 11-12 janvier, 7-18.

13. Pautrizel (R.), Tribouley (J.), Szersnovicz (F.) et Tribouley-Duret (J.), 1968. Déductions à tirer d'une enquête séro-immunologique faite aux Antilles françaises. Bordeaux Méd., 10, 1765-1767.

14. Pettersen (E. K.), 1968. - Prepa-ation of Toxoplasma gondii antigen for the complement fixation test. Acta path. microbiol. scand., 74, 35-40.

15. Quilici (M.), Ranque (Ph.), Tounkara (A.) et Rougemont (A.), 1976. - La toxoplasmose en République du Mali. Approche épidémiologique. Acta tropica, 33, 229-239.

16. Remington (J. S.), Efran (B.), Cavanangh (E.), Simar (H. J.) et Trejos (A.), 1970. Studies on toxoplasmosis in El Salvador. Prevalence and incidence of toxoplasmosis as measured by the Sabin-Feldman dye-test. Trans. R. Soc. trop. Med. Hyg., 64, 252-267.

17. Seguela (J.-P.), Larrouy (G.), Sérié (Ch.) et Plenet (J.), 1975. - Toxoplasmose en Guyane française. Med. Mal. infect., 5, 546-548.

18. Tribouley (J.), Tribouley-Duret (J.), Iribarren (M.-J.) et Pautrizel (R.), 1969. Mise en évidence des anticorps spécifiques de Fasciola hepatica par hémagglutination passive en utilisant le glutaraldéhyde comme agent de couplage. C.R. Acad. Sci. (Paris), 268, 2215-2217. 
19. Tribouley (J.), Capbern (A.) et Bezian (J.-H.), 1971. - Mise en évidence d'anticorps antithyroïdiens à l'aide d'une réaction d'hémagglutination passive utilisant le glutaraldéhyde comme agent de couplage. C.R. Acad. Sci. (Paris), 272, 1462-1464.

20. Tribouley (J.), Etcharry (G.), Etcharry (F.) et Pautrizel (R.), 1971. - Intérêt d'une réaction d'hémagglutination passive pour le sérodiagnostic de la toxoplasmose. Comptes Rendus $1^{\text {er }}$ Multicolloque Européen de Parasitologie, Rennes, 1-4 septembre, 267-269.

21. Tribouley (J.), Tribouley-Duret (J.), Bernard (D.), Appriou (M.) et Pautrizel (R.), 1975. - La bilharziose intestinale en Guadeloupe. Bull. Soc. Pathol. exot., $68,180-193$.

22. Wallace (G. D.), 1976. - The prevalence of toxoplasmosis on Pacific islands and the influence of ethnic group. Am. J. Trop. Med. Hyg., 25, 48-53.

23. Walls (K. W.), Kagan (I. G.) et Turner (A.), 1967. - Studies of the prevalence of antibodies to Toxoplasma gondii. 1. - U.S. Military recruits. Am. J. Epidem., 85, 87-92.

24. Walls (K. W.) et Kagan (I. G.), 1967. - Studies on the prevalence of antibodies to Toxoplasma gondii. 2. - Brazil. Am. J. Epidem., 86, 305-313.

25. Work (K.), 1971. - Toxoplasmosis with special reference to transmission and life cycle of Toxoplasma gondii. Acta Pathol. Microbiol. Scand., Sect. B, Suppl. 221, 7, 41.

26. Work (K.) et Hutchinson (W. M.), 1969. - A new cystic form of Toxoplasma gondii. Acta Pathol. Microbiol. Scand., 75, 191-192. 